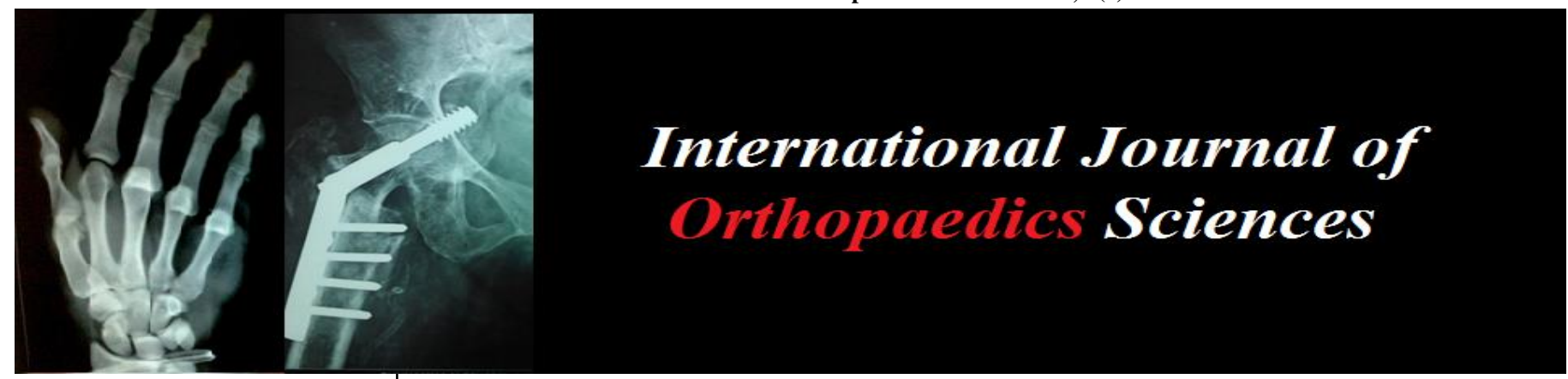

E-ISSN: 2395-1958

P-ISSN: 2706-6630

IJOS 2021; 7(3): 37-41

(C) 2021 IJOS

www.orthopaper.com

Received: 28-07-2021

Accepted: 13-09-2021

Dr. Vinay Vasu Bangera Assistant Professor, M.S Orthopaedics, Dept of Orthopaedics AJ Institute of Medical Sciences, Mangalore Karnataka, India

Dr. Naimish Hegde

Junior Resident, M.S

Orthopaedics, Dept of

Orthopaedics, AJ Institute of

Medical Sciences, Mangalore,

Mangalore, Karnataka, India

Dr. Kaveesh S Shetty

Junior Resident, M.S

Orthopaedics, Dept of

Orthopaedics, AJ Institute of

Medical Sciences, Mangalore,

Karnataka, India

Dr. Shree Krishnananda Sagar

Junior Resident, M.S

Orthopaedics, Dept of

Orthopaedics, AJ Institute of

Medical Sciences, Mangalore,

Karnataka, India
Corresponding Author: Dr. Naimish Hegde Junior Resident, M.S Orthopaedics, Dept of Orthopaedics, AJ Institute of Medical Sciences, Mangalore, Mangalore, Karnataka, India

\section{Latissimus dorsi tendon transfer for massive unrepairable rotator cuff tears: A case report}

\author{
Dr. Vinay Vasu Bangera, Dr. Naimish Hegde, Dr. Kaveesh S Shetty and \\ Dr. Shree Krishnananda Sagar
}

DOI: https://doi.org/10.22271/ortho.2021.v7.i4a.2864

\section{Abstract}

Introduction: Massive and irreparable posterior-superior rotator cuff tears present a difficult treatment problem. Chronic rotator cuff tears can cause tendon retraction and fatty atrophy of the muscles. Fullthickness rotator cuff tears involves more than one tendon cause superior migration of the humeral head in relation to the glenoid with altered glenohumeral forces, and ultimately leading to rotator cuff arthropathy. Transfer of the latissimus dorsi muscle is considered a surgical option in the treatment of younger patients without glenohumeral arthritis and with functional deficits caused by an irreparable posterior-superior rotator cuff tear.

Presentation of Case: This report presents a case of Right Supraspinatus and Infraspinatus Chronic tear with Subscapularis tear. The case was chronic with non-subsidence of symptoms in spite of conservative treatment. Our treatment in this case consisted of Right Subscapularis and Infraspinatus Repair with Lattisimus Dorsi Transfer to Greater Tubercle. Post operatively ultrasling was used to keep the shoulder in neutral with slight abduction. Rehabilitation protocol was begun after 3 weeks of immobilization.

Discussion: Latissimus Dorsi Tendon Transfer has been considered to be a means of restoring function and providing pain relief in patients with irreparable tears involving the infraspinatus and supraspinatus tendons in the absence of substantial glenohumeral arthritis.

The aim of this case presentation is to report an example for this case, highlight the difficulties in diagnosis and the treatment options.

Conclusion: The Latissimus Dorsi Tendon Transfer for irreparable postero-superior cuff deficiency in selected patients reduce pain and improve shoulder function in the medium term.

Keywords: Hip fracture, Bone turnover markers, CTX, PINP, Vitamin D

\section{Introduction}

Massive irreparable posterior-superior rotator cuff tears present a difficult treatment problem. Chronic rotator cuff tears can cause tendon retraction and fatty atrophy of the muscles. Fullthickness rotator cuff tears involving more than one tendon cause superior migration of the humeral head in relation to the glenoid with altered glenohumeral forces, and ultimately leading to rotator cuff tear arthropathy ${ }^{[1,4]}$.

Hemiarthroplasty ${ }^{[5,6]}$ components designed to compensate for rotator cuff tear arthropathy provide pain relief but are less reliable for restoring shoulder function in patients with irreparable tears of two or more tendons. Reverse total shoulder arthroplasty ${ }^{[7,9]}$ is indicated for low-demand elderly patients with rotator cuff arthropathy, restores function in addition to providing pain relief.

Patients with glenohumeral joint arthropathy from an irreparable rotator cuff tear are not generally treated with tendon transfer as this treatment option does not address the joint degeneration. However, one study ${ }^{[10]}$ demonstrated good results with use of the reverse total shoulder prosthesis in patients with an irreparable rotator cuff tear and no glenohumeral arthritis. Despite this, there are concerns regarding the longevity of reverse total shoulder implants and limited possibilities for salvage after implant failure. As a result, transfer of the latissimus dorsi muscle is considered a surgical option in the treatment of younger patients without glenohumeral arthritis and with functional deficits caused by an irreparable posteriorsuperior rotator cuff tear. 
However, the results and expectations for this procedure have been reported to be variable ${ }^{[11,23]}$.

Latissimus dorsi transfer provides a large, vascularized tendon that closes the cuff defect and exerts an external rotational moment, allowing more effective action of the deltoid muscle. In its native location, the latissimus dorsi muscle contributes to internal rotation, retroversion, and abduction of the shoulder joint. When the latissimus dorsi tendon is transferred to the greater tuberosity, the muscle's internal rotator torque is removed and the function of the muscle changes into an external rotator ${ }^{[24,25]}$. This external rotation is accomplished by either a synergistically active tendon transfer or a tenodesis effect ${ }^{[16]}$.

Electromyographic studies have suggested that in some cases the transfer is truly active whereas in others the patient cannot actively synchronize latissimus dorsi muscle activity with supraspinatus and infraspinatus muscle activity [11, 18, 26]. In either case, the improved balance between the anterior and posterior soft tissue structures of the shoulder is believed to be biomechanically important to serve as a balanced fulcrum as described by Burkhart [27]. Furthermore, this balance across the glenohumeral joint is believed to allow the deltoid muscle to contribute to shoulder motion more effectively [27].

\section{Case Report}

49 year old female patient presented with complaints of pain in the right shoulder since 6 months following an alleged history of slip and fall at her residence 1 year back. Patient was managed conservatively with analgesics and physiotherapy in the local hospital. In view of persisting pain and difficulty in doing daily activities in spite of conservative treatment patient was advised MRI of Right Shoulder. Imaging done confirmed the diagnosis of Right Supraspinatus and Infraspinatus Chronic tear with Subscapularis tear. Patient was later referred to tertiary care hospital for further management.

On examination, we found that there was restriction of range of movements and there was no evidence of peripheral nerve injuries. There were no associated injuries in other extremities.
ASES scores were assessed pre and post operatively.

\section{Pre-Operative Range of Motion}

Flexion: 0-40

Extension: 0-30

Internal Rotation: $0-70^{\circ}$

External Rotation: 0-40

Adduction: $0-30^{\circ}$

Abduction: $0-80^{\circ}$

ASES Score: 28.3

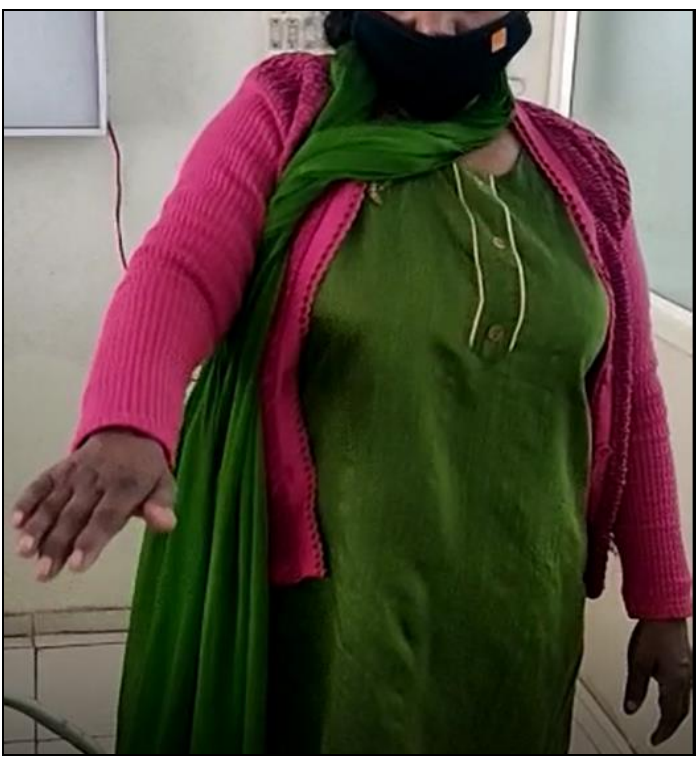

\section{MRI scan of right shoulder}

MRI scan was re-evaluated with the help of the radiologist to note the following findings with assessment of severity based on Goutallier Classification

Complete thickness tear of right supraspinatus, infraspinatus tendon at their humeral attachment with retraction and severe atrophy - Chronic tear (Grade 4 according to Goutallier Classification)

\section{Acromio Clavicular Joint arthritis Right Shoulder joint effusion}

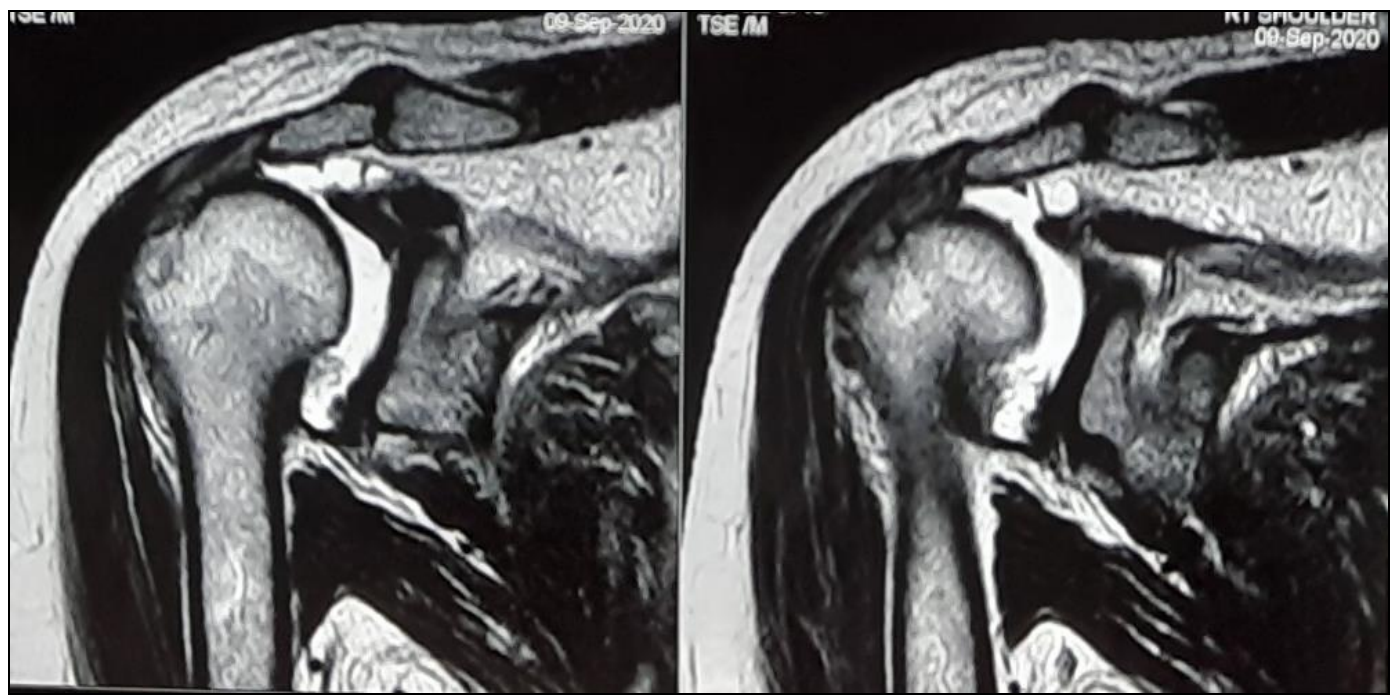



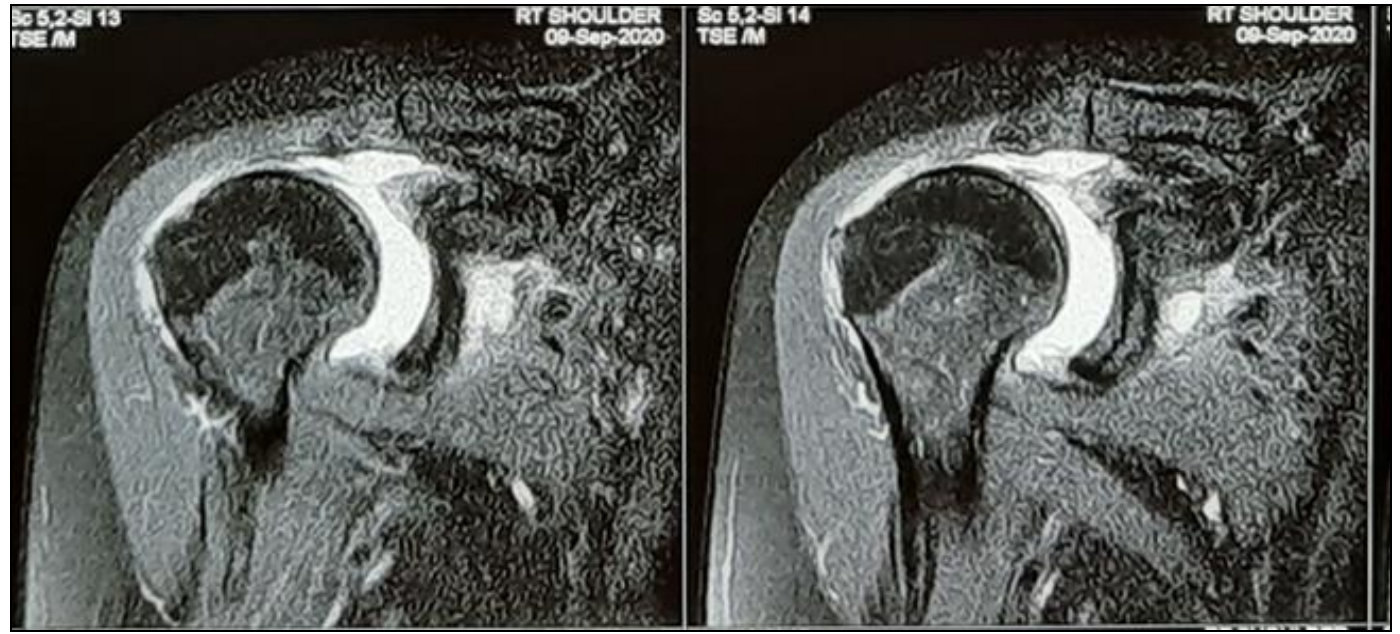

\section{Surgical Procedure}

Patient underwent Right Subscapularis and Infraspinatus Repair with Latissimus Dorsi Transfer to Greater Tubercle Fig (1-5) with Acromioplasty in AJ Institute of Medical Sciences and Research Centre, Mangalore.

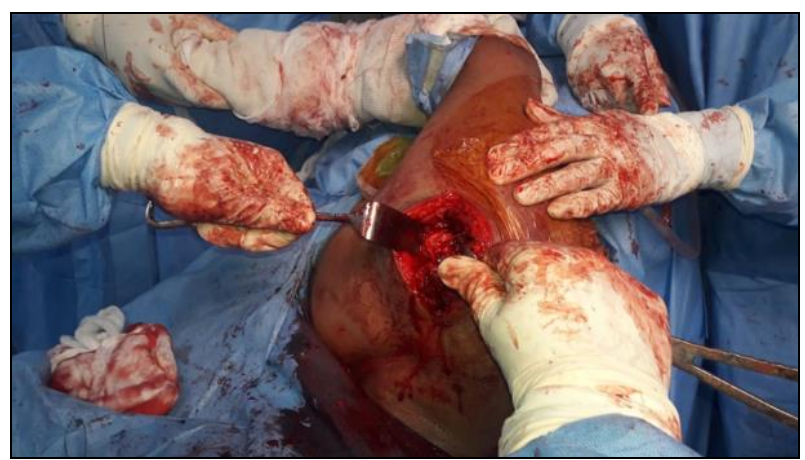

Fig 1: Dissection done upto visualisation of rotator cuff muscles

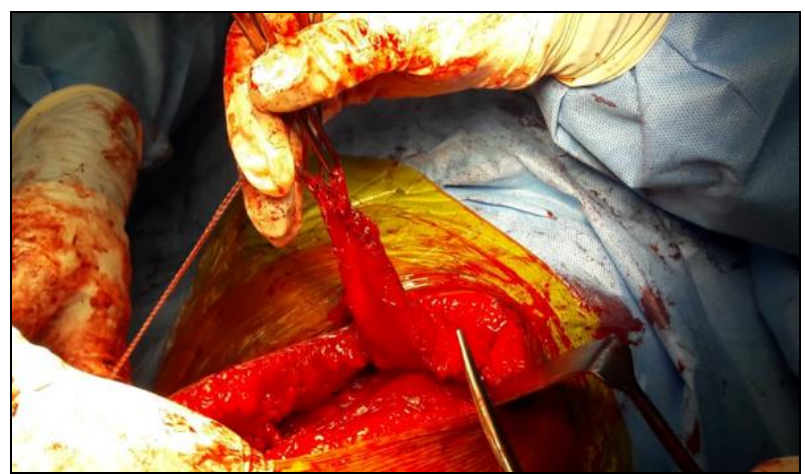

Fig 2: Latissimus dorsi tendon released from its attachment and secured with suture

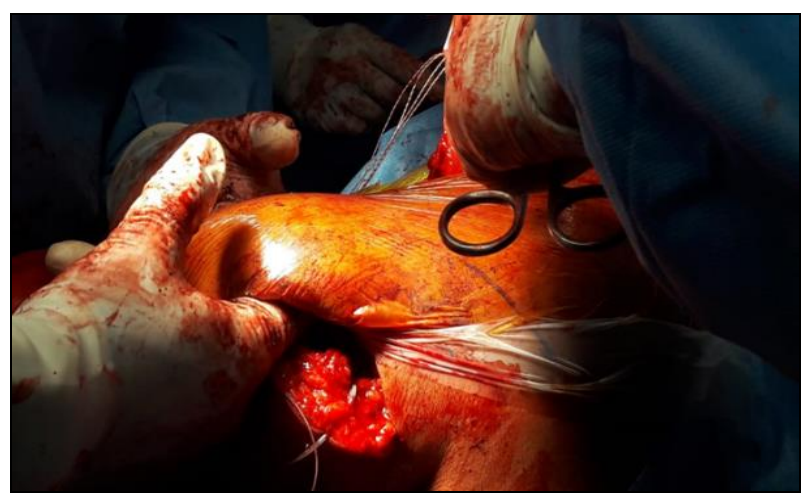

Fig 3: Tunnel deep to the deltoid and superficial to the infraspinatus and teres major is made

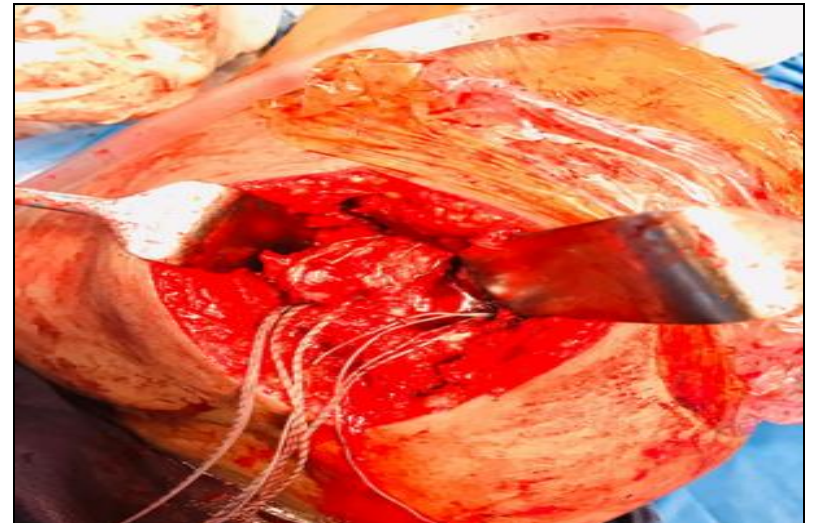

Fig 4: The tendon has been secured to the remaining portions of the infraspinatus and supraspinatus tendons with non-absorbable sutures

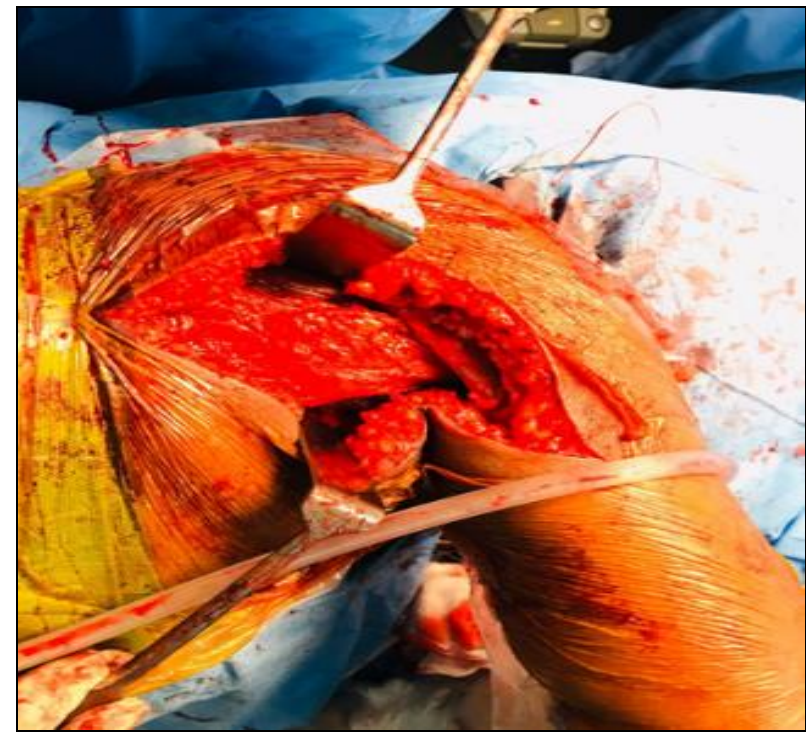

Fig 5: The lateral portion of the latissimus tendon has been secured to the greater tuberosity with non-absorbable sutures that were passed through the bone

\section{Post-Operative Protocol}

Post operatively, right shoulder was kept in neutral position and slight abduction with elbow in 90 degree flexion using Ultrasling.

\section{Follow Up}

On $8^{\text {th }}$ post-operative day, patient was discharged. Skin staples were removed after 2 week postoperatively. Physiotherapy was started after 3 weeks of immobilization with only passive movements in the plane of scapula i.e, 
Passive shoulder flexion upto 90 degrees, Passive pendular exercises, Scapular protraction and retraction exercises. Patient was reviewed after 8 weeks, was admitted for active physiotherapy. TENS and retraining therapy was given to improve function of Latissimus Dorsi.

\section{Post-operative range of motion (6 months follow up)}

Flexion: 0-130

Extension: 0-30

Internal Rotation: $0-70^{\circ}$

External Rotation: 0-40

Adduction: 0-30

Abduction: $0-80^{\circ}$

ASES Score: 68.3

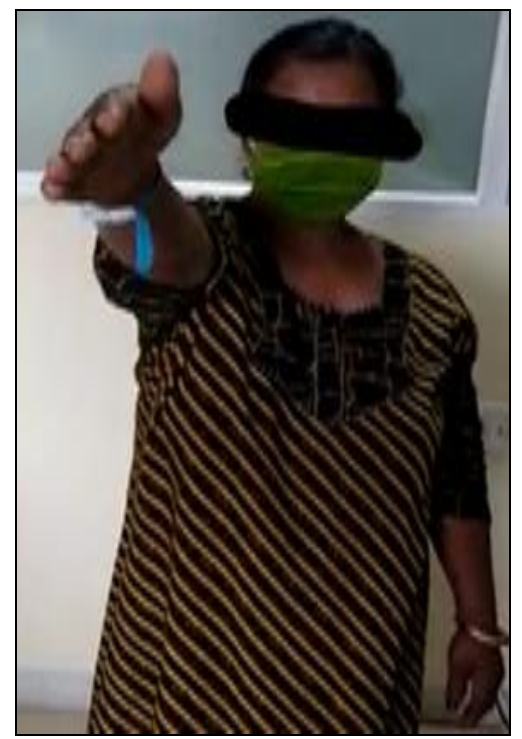

Fig 6: 6 months follow up

\section{Discussion}

Latissimus Dorsi Tendon Transfer has been considered to be a means of restoring function and providing pain relief in patients with irreparable tears involving the infraspinatus and supraspinatus tendons in the absence of substantial glenohumeral arthritis.

Latissimus Dorsi Tendon Transfer to the greater tuberosity has been the most successful tendon transfer procedure for irreparable supraspinatus and infraspinatus tears associated with severe functional impairment and chronic pain.

Gerber et al. ${ }^{[26]}$ introduced this idea using a two incision technique - a dorsal incision to mobilize latissimus dorsi and a second, transacromial approach to provide access to the greater tuberosity of the humerus.

Aoki et al. ${ }^{[11]}$ published the results of 12 Latissimus Dorsi Tendon Transfer with a follow-up of 35.6 months. Eight patients were reported to have excellent or good results and four patients had fair or poor results.

Principles of tendon transfers around the shoulder are as follows:

- Shoulder elevation is most efficiently performed in the plane of the scapula; tendon transfers to restore shoulder elevation should therefore be performed in the plane of the scapula when possible.

- Tendons transferred to the humeral head (or rotator cuff) in order to bring about rotation are more efficient than transfers to the shaft of the humerus as the humeral head has a greater diameter.
Miniaci and MacLeod ${ }^{[20]}$ followed up 17 patients who were managed with an LDTT as a salvage procedure; 14 gained significant pain relief and an improvement in shoulder function and 3 were classed as a failure with ongoing pain and impaired function.

Iannotti et al. ${ }^{[18]}$ found female sex, generalized muscle weakness and poor preoperative shoulder function to be negative predictors of outcome. They also noted that poor electrical activity of the Latissimus Dorsi Tendon Transfer at follow-up was a negative factor.

Some authors advocate the use of both the teres major tendon and the latissimus dorsi tendon in order to increase the amount of tendon tissue available for coverage of the posterosuperior cuff footprint. This combined transfer has been used extensively for the treatment of Erb's palsy in the pediatric population and is supported by biomechanical studies ${ }^{[25,28,29]}$. An anatomical study by Herzberg et al.(30) showed that reinsertion of the latissimus dorsi flap at the site of insertion of infraspinatus gave superior rotational movement compared with that of insertion at other sites.

Moursey et al. ${ }^{[21]}$ showed superior results with a modified tendon transfer technique. They harvested the tendon with a small piece of bone, which then allowed bone-to-bone transosseous fixation. Less ruptures were seen on MRI when assessing the integrity of the transfer at follow-up. This correlated with an improvement in patient satisfaction.

\section{Conclusion}

Latissimus Dorsi Tendon Transfer significantly improve pain and function in young patients with postero-superior rotator cuff deficiency. It is successful when primary repair of the rotator cuff proves impossible, or as a salvage procedure. The procedure is reproducible and in the medium term has good results. The authors believe Latissimus Dorsi Tendon Transfer should not be forgotten as an alternative in the management of massive rotator cuff deficiency, rather than opting for a reverse polarity total shoulder replacement in the non-arthritic shoulder.

\section{References}

1. Feeney MS, O'dowd J, Kay EW, Colville J. Glenohumeral articular cartilage changes in rotator cuff disease. J Shoulder Elbow Surg 2003;12:20-3.

2. Hamada K, Fukuda H, Mikasa M, Kobayashi Y. Roentgenographic findings in massive rotator cuff tears. A long-term observation. Clin Orthop Relat Res 1990;254:92-6.

3. Neer CS, Craig EV, Fukuda H. Cuff-tear arthropathy. J Bone Joint Surg Am 1983;65:1232-44.

4. Loehr JF, Helmig P, Søjbjerg JO, Jung A. Shoulder instability caused by rotator cuff lesions. An in vitro study. Clin Orthop Relat Res 1994;304:84-90.

5. Goldberg SS, Bell JE, Kim HJ, Bak SF, Levine WN, Bigliani LU. Hemiarthroplasty for the rotator cuffdeficient shoulder. J Bone Joint Surg Am 2008;90:554-9.

6. Sanchez-Sotelo J, Cofield RH, Rowland CM. Shoulder hemiarthroplasty for glenohumeral arthritis associated with severe rotator cuff deficiency. J Bone Joint Surg Am 2001;83:1814-22.

7. Cuff D, Pupello D, Virani N, Levy J, Frankle M. Reverse shoulder arthroplasty for the treatment of rotator cuff deficiency. J Bone Joint Surg Am 2008;90:1244-51.

8. Matsen FA, Boileau P, Walch G, Gerber C, Bicknell RT. The reverse total shoulder arthroplasty. J Bone Joint Surg Am 2007;89:660-7. 
9. Wall B, Nov-Josserand L, O'Connor DP, Edwards TB, Walch G. Reverse total shoulder arthroplasty: a review of results according to etiology. J Bone Joint Surg Am 2007;89:1476-85.

10. Mulieri P, Dunning P, Klein S, Pupello D, Frankle M. Reverse shoulder arthroplasty for the treatment of irreparable rotator cuff tear without glenohumeral arthritis.J Bone Joint Surg Am 2010;92:2544-56.

11. Aoki M, Okamura K, Fukushima S, Takahashi T, Ogino T. Transfer of latissimus dorsi for irreparable rotator-cuff tears. J Bone Joint Surg Br 1996;78:761-6.

12. Codsi MJ, Hennigan S, Herzog R, Kella S, Kelley M, Leggin B, Williams GR, Iannotti JP. Latissimus dorsi tendon transfer for irreparable posterosuperior rotator cuff tears. Surgical technique. J Bone Joint Surg Am. 2007;89(Suppl 2):1-9.

13. Costouros JG, Espinosa N, Schmid MR, Gerber C. Teres minor integrity predicts outcome of latissimus dorsi tendon transfer for irreparable rotator cuff tears. J Shoulder Elbow Surg 2007;16:727-34.

14. Degreef I, Debeer P, Van Herck B, Van Den Eeden E, Peers K, De Smet L.Treatment of irreparable rotator cuff tears by latissimus dorsi muscle transfer. Acta Orthop Belg 2005;71:667-71.

15. Gerber C. Latissimus dorsi transfer for the treatment of irreparable tears of the rotator cuff. Clin Orthop Relat Res 1992;275:152-60.

16. Gerber C, Maquieira G, Espinosa N. Latissimus dorsi transfer for the treatment of irreparable rotator cuff tears. J Bone Joint Surg Am 2006;88:113-20.

17. Gerhardt C, Lehmann L, Lichtenberg S, Magosch P, Habermeyer P. Modified L'Episcopo tendon transfers for irreparable rotator cuff tears: 5-year follow-up. ClinOrthop Relat Res. 2010;468:1572-7.

18. Iannotti JP, Hennigan S, Herzog R, Kella S, Kelley M, Leggin B, Williams GR. Latissimus dorsi tendon transfer for irreparable posterosuperior rotator cuff tears. Factors affecting outcome. J Bone Joint Surg Am 2006;88:342-8.

19. Irlenbusch U, Bracht M, Gansen HK, Lorenz U, Thiel J. Latissimus dorsi transfer for irreparable rotator cuff tears: a longitudinal study. J Shoulder Elbow Surg 2008; 17:527-34.

20. Miniaci A, MacLeod M. Transfer of the latissimus dorsi muscle after failed repair of a massive tear of the rotator cuff. A two to five-year review. J Bone Joint Surg Am 1999;81:1120-7.

21. Moursy M, Forstner R, Koller H, Resch H, Tauber M. Latissimus dorsi tendon transfer for irreparable rotator cuff tears: A modified technique to improve tendon transfer integrity. J Bone Joint Surg Am 2009;91:192431.

22. Nov'-Josserand L, Costa P, Liotard JP, Safar JF, Walch $\mathrm{G}$, Zilber S. Results of latissimus dorsi tendon transfer for irreparable cuff tears. Orthop Traumatol Surg Res 2009;95:108-13.

23. Tauber M, Moursy M, Forstner R, Koller H, Resch H. Latissimus dorsi tendon transfer for irreparable rotator cuff tears: a modified technique to improve tendon transfer integrity: surgical technique. J Bone Joint Surg Am 2010;92(Suppl 1):226-39

24. Bogduk N, Johnson G, Spalding D. The morphology and biomechanics of latissimus dorsi. Clin Biomech (Bristol, Avon) 1998;13:377-85.

25. Magermans DJ, Chadwick EK, Veeger HE, Rozing PM, van der Helm FC. Effectiveness of tendon transfers for massive rotator cuff tears: a simulation study. ClinBiomech (Bristol, Avon) 2004;19:116-22.

26. Gerber C, Vinh TS, Hertel R, Hess CW. Latissimus dorsi transfer for the treatment of massive tears of the rotator cuff. A preliminary report. Clin Orthop Relat Res 1988;232:51-61.

27. Burkhart SS. Arthroscopic treatment of massive rotator cuff tears. Clinical results and biomechanical rationale. Clin Orthop Relat Res 1991;267:45-56.

28. Edwards TB, Baghian S, Faust DC, Willis RB. Results of latissimus dorsi and teres major transfer to the rotator cuff in the treatment of Erb's palsy. J Pediatr Orthop 2000;20:375-9

29. L'Episcopo JB. Tendon transplantation in obstetric paralysis. Am J Surg 1934;25:122-5.

30. Herzberg G, Schoierer O, Berthonnaud E, Medda N, Dimnet J. 3D mdelization of latissimus dorsi transfer in rotator cuff surgery: what point of fixation on the humeral head. 1st Closed Meeting European Society Surgery Shoulder Elbow 2001. 